\title{
Is an Interventional Oncology Center an advantage in the service of cancer patients or in the education? The Gemelli Hospital and INTERACTS experience
}

\author{
Prof. György Kovács, MD, PhD 1,2, Luca Tagliaferri, MD, PhD 3.4 , Prof. Vincenzo Valentini, MD, PhD5,6 \\ Interdisciplinary Brachytherapy Unit, University of Lübeck/UKSH-CL, Germany, ${ }^{2}$ INTERACTS Scientific Program Director, ${ }^{3}$ Polo Scienze \\ Oncologiche ed Ematologiche, Università Cattolica del Sacro Cuore, Fondazione Policlinico Universitario Agostino Gemelli, Roma, Italy, \\ ${ }^{4}$ INTERACTS courses organizer, ${ }^{5}$ Polo Scienze Oncologiche ed Ematologiche, Istituto di Radiologia, Università Cattolica del Sacro Cuore. \\ Fondazione Policlinico Universitario Agostino Gemelli, Roma, Italy, ${ }^{6}$ INTERACTS School Director
}

Address for correspondence: Prof. György Kovács, MD, PhD, e-mail: kovacsluebeck@gmail.com

Modern oncology needs to offer personalized treatments. The process of choosing the best possible treatment should be based on efficacy, feasibility, and cost benefit, but also considering the patient's age, clinical condition, presentation of disease, and the patient's individual needs (travel, work, family, etc.). Interdisciplinarity is playing a major role in the diagnostic workout and in the treatment decision, as well as in the performance and effectiveness of the procedures. Minimal invasive procedures are preferred, but the performance needs highly specialized teams.

Treatment decisions usually depend on 'through which door the patient enters', i.e. which specialist has the first contact with the patient. Frequently, anatomic siterelated multidisciplinary tumor boards (MDT) are in lack of experts of all kind of interventional procedures. Therefore, the idea was born to create a platform directly linked to the anatomic site-related MDT's. Interventional expert knowledge was collected under the name of 'interventional oncology', in order to connect highly specialized technology, anatomic site related MDT knowledge, as well education (INTERACTS) and research (COBRA) in the field. Four main interventional fields were identified:

1) interventional radiology: treatment/procedure performed under image guidance without the use of radioisotopes like MWA (microwave ablation), RFA (radiofrequency ablation), TAE (transcatheter arterial embolization), biopsy, positioning of fiducials, and/or catheters and stents;

2) interventional radiotherapy: treatment/procedure using radioisotopes like brachytherapy or TARE (transarterial radioembolization);

3) interventional chemotherapy: treatment/procedure using focused chemotherapy like TACE (transarterial chemoembolization), electrochemotherapy;

4) interventional endoscopy: treatment/procedure using an endoscopic approach like PEG (percutaneous gastrostomy), biopsy, positioning of fiducials, and/or catheters and stents.
Many procedures could be equally introduced among the above interventional fields, and only a detailed multidisciplinary case evaluation and discussion could be offered to the patient as the best therapeutic method. This complex procedure needs eligible physicians working together and in close cooperation with other institutional MDT's; an Interventional Oncology Center (IOC) can offer a possible solution. By definition, the IOC is an 'interdisciplinary service for diagnosis and treatment of cancer and cancer-related problems using targeted (focal) minimally invasive procedures'. The IOC should have the possibility to evaluate every patient by a radiation oncology expert with experience in interventional procedures, an interventional radiology, and an interventional endoscopy specialist. The radiation oncologist is involved in interventional radiotherapy procedures as well in treatments linked with external beam radiotherapy (EBRT), such as the positioning of prostate fiducials or combining EBRT and IRT and/or surgery.

The new Interventional Oncology Center at the Catholic University, Agostino Gemelli Hospital in Rome, was opened in November during the $5^{\text {th }}$ GEC-ESTRO workshop (Figures 1 and 2). The Center is a GEMELLI-ART (Advanced Radiation Therapy) service, and is connected with the interventional radiology service as well as with the endoscopic service. Every week, cancer patients who are candidates for minimally invasive procedures as a monotherapy or as part of a combined procedure involving interventional therapy techniques, are discussed in a multidisciplinary board. The radiation oncologist, the interventional radiologist, and the interventional endoscopy expert attend at the 'INTER-BOARD' (INTERventional and focal treatment BOARD), and the treatment decision is documented as a report in a written form.

The Interventional Oncology Center has a dedicated area with two shielded application rooms, a treatment planning and clinical dosimetry room, an outpatient 


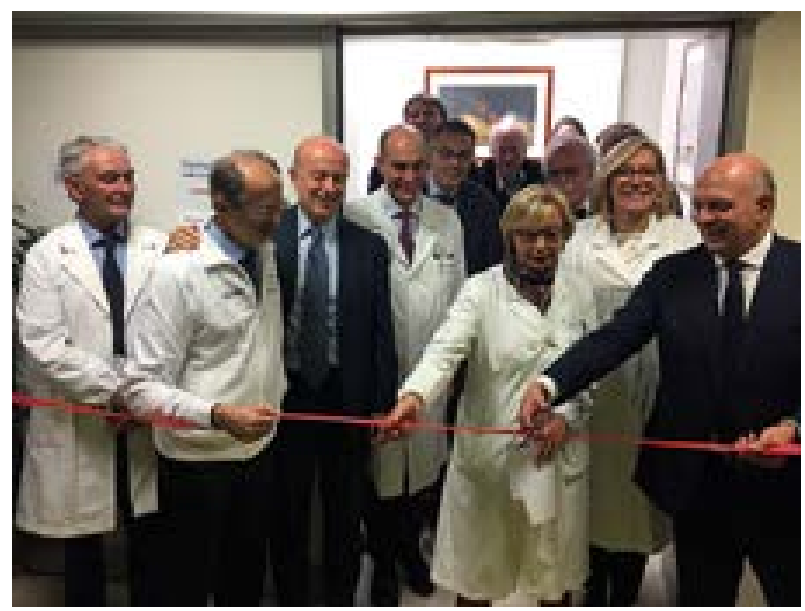

Fig. 1. Cutting the ribbon at the opening ceremony of the Gemelli IOC

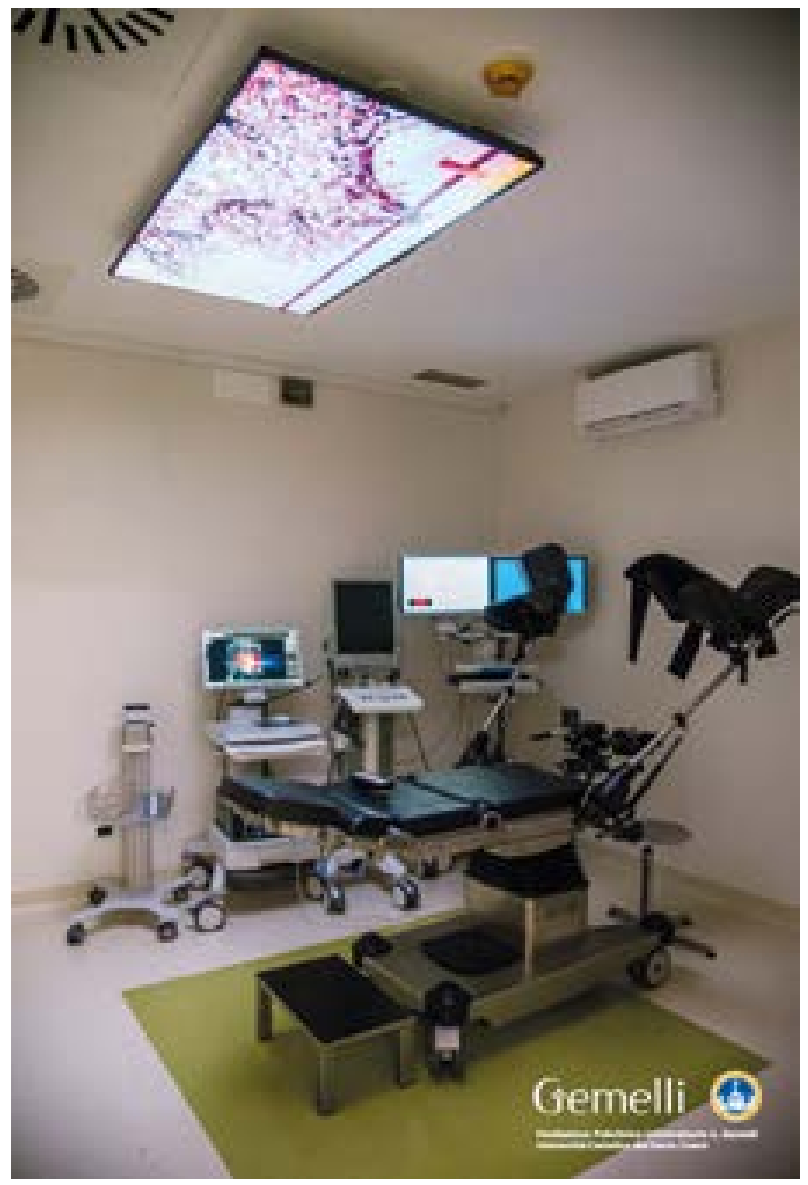

Fig. 2. One of the IOC treatment rooms

room, and other areas for personnel, as well as for source storage and manipulation. In the GEMELLI ART - IOC, interventional radiotherapy procedures (IRT, often called brachytherapy), electrochemotherapy, and fiducial positioning procedures can be performed by the use of imageguided techniques with or without image fusion software solutions and navigation systems.

Nowadays, IRT plays increasingly important role in cancer treatments supported by impressive progress of 3D imaging, rapid growth of capacity and speed of computers, and sophisticated treatment planning techniques. The dose modulation potential paired with biological target imaging information opened a new era; therefore, there is a necessity for education regarding these techniques. For this reason in GEMELLI ART - IOC, an Interventional Radiotherapy School was founded, the GEMELLI-INTERACTS (INTERventional Radiotherapy ACtive Teaching School). The school is a 'Gemelli ART' service and the name means: 'Catholic University' by Agostino Gemelli Hospital interacts with physicians and physicist interested in education and research in interventional radiotherapy. The mission is the education of physicians/medical physicists in interventional radiotherapy (brachytherapy) through a multidisciplinary approach with small group (max. 20 participants) theoretical lessons and practical exercises in the operation rooms of different specialties, as well as in the IOC.

Furthermore, the GEMELLI ART - IOC is connected and supported by a 'Knowledge Based Laboratory' that is using the data of large databases (COBRA - COnsortium for BRAchytherapy) to create predictive models, which could be useful in the clinical practice. The head \& neck and skin GEC-ESTRO Working Group support the work with these large databases. At the moment, 18 centers worldwide joined the consortium for sharing the data in head and neck, eye, and skin patient cohorts.

\section{References}

1. Tagliaferri L, Pagliara MM, Boldrini L et al. INTERACTS (INTErventional Radiotherapy ACtive Teaching School) guidelines for quality assurance in choroidal melanoma interventional radiotherapy (brachytherapy) procedures. J Contemp Brachytherapy 2017; 9: 287-295.

2. Tagliaferri L, Kovács G, Autorino R et al. ENT COBRA (Consortium for Brachytherapy Data Analysis): interdisciplinary standardized data collection system for head and neck patients treated with interventional radiotherapy (brachytherapy). J Contemp Brachytherapy 2016; 8: 336-343. 